\title{
28 \\ CAC investigation for video and data
}

\author{
E.Aarstad ${ }^{a}$, S.Blaabjerg ${ }^{b}$, F.Cerdan $^{c}$, S.Peeters ${ }^{d}$ and \\ K.Spaey ${ }^{d}$
}

${ }^{a}$ Telenor Research \& Development, P.O. Box 83, N-2007

Kjeller, Norway,egil.aarstad@fou.telenor.no

${ }^{b}$ Tele Denmark, Telegade 2, DK-2630 Taastrup, Denmark, sblb@dtk.dk

${ }^{c}$ Polytechnic University of Catalonia, Campus Nord. Modulo D6, E-08071 Barcelona, Spain, fernando@ac.upc.es

${ }^{d}$ University of Antwerp, Universiteitsplein 1, B-2610 Antwerp, Belgium, \{speeters,spaey\}@uia.ua.ac.be

\begin{abstract}
A key objective of ATM-based networks is to provide at the same time guaranteed QoS to real time and non-real time services. This calls for thoroughly engineered traffic control methods for those service categories as well as for the overall integration strategy. The main objective of this paper is to investigate CAC in an ATM testbed with real switches and as realistic traffic as possible. The used traffic is video (MPEG model based on traces) and data modelled as traditional on/off sources. The video traffic is given priority over the data traffic. To complement and verify the experiments, two simulation tools using as input the artificial MPEG models and the real traces have been developed. The CAC boundary for the nonpriority case has also been derived analytically. The experimental sources are modelled as discrete-time Markov sources. A matching method to avoid state space explosion for the superposition is applied on the source models.
\end{abstract}

\section{Keywords}

Connection Admission Control (CAC), delay priorities, multiplexing experiments 


\section{INTRODUCTION}

One of the most important objectives of ATM is the integration of different services into one multi-service network while providing differentiated Quality of Service (QoS). In order to meet this objective, an integrated traffic control framework which can give performance guarantees while ensuring efficient network utilization is required.

An important ATM traffic control function is Connection Admission Control (CAC). CAC determines whether a new connection can be accepted or not, depending on the availability of the necessary resources. Since the traffic types differ not only in their peak and mean rate but also in their burstiness, the CAC function has to be designed carefully to make correct bandwidth allocation decisions in order to avoid wasting bandwidth.

With the increasing interest in multimedia and the tremendous growth in the Internet traffic it is expected that a significant part of the future broadband traffic will consist of video as well as data traffic. Most literature on video and data so far considers statistical multiplexing (by simulation and analytically) of video and data separately, see e.g. (Maglaris, 1988), (Reininger, 1994), (Rose, 1997), (Roberts, 1996) and (Leland, 1994).

In this paper we investigate a CAC solution with delay priorities for integrating real time and non-real time Variable Bit Rate (rt-VBR and nrt-VBR) services. We present results from experiments performed within the framework of the ACTS (Advanced Communications Technologies and Services) EXPERT project at the EXPERT Testbed in Basle, Switzerland.

Section 2 presents an artificial MPEG traffic model and the on/off data model, a description of the configuration and the obtained CAC boundaries. Additional to the experiments a simulator has been developed. The results of the simulations and a comparison with the experimental results is also given in section 2. Section 3 presents a tool which enables a study of multiplexing the real traces and on/off data sources. In section 4, a CAC boundary for the non-priority case is analytically derived by describing the sources used in the experiments as Discrete-time Batch Markovian Arrival Processes (D-BMAPs) and approximating their superposition by a new D-BMAP.

\section{EXPERIMENTAL CAC BOUNDARIES}

\subsection{Modelling video and data}

In order to reduce the required bandwidth to acceptable levels, several coding schemes for the compression of video streams have been developed, with MPEG as the most promising scheme. It comes in two versions, MPEG-I (see (Le Gall, 1991) and (ISO/IEC, 1993a)) and MPEG-II (see (ISO/IEC, 1993b)) where the MPEG-I functionality is a subset of that of MPEG-II. In this paper it is MPEG-I which is dealt with. MPEG encoding of a video sequence is realised using three different 


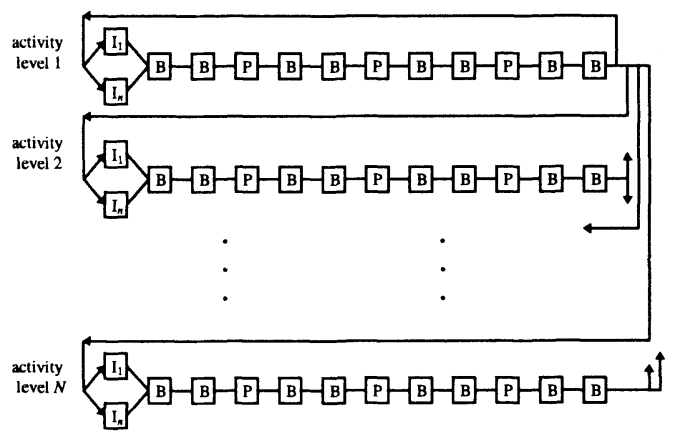

Figure 1: Structure of the artificial MPEG source

compression levels. Thereby three different frame types, I-, P- and B, are generated. A periodic generation of a pattern of these frame types is the result.

From a traffic modelling point of view, MPEG has traditionally either been modelled as Markovian (Rose, 1997), autoregressive (Ramamurthy, 1992) or more recently fractal (Beran, 1995). Since the traffic generators available at the EXPERT platform implement a Markovian model at the burst level, we have chosen to follow the Markovian approach of B. Helvik (Helvic, 1993). No intention to capture long range correlation is made, mainly due to our multiplexing scenarios where only short or moderate sized buffers are used. The periodic generation is compromised in the sense that the duration in individual states is assumed exponential instead of constant (a requirement if more than one MPEG source on each Synthesized Traffic Generator (STG) is to be generated). The sum for B- and P-frames is approximated by using four or five load levels and the I-frame size distribution is further refined by defining two sublevels within each load level (see Figure 1). The duration of each frame is $45 \mathrm{msec}$. Parameters in the model are the transition probabilities between the load levels as well as the I, B and P rate at each load level. A method developed by B. Helvik (and quite similar to the simple Markov method presented in the video modelling section in part I of (Roberts, 1996)) to generate these parameters from an arbitrary MPEG video trace has been used. For more details we refer to (Helvic, 1996). Using artificial MPEG sources enables us to investigate traffic scenarios with more sources than would have practically possible using only real MPEG sources and makes our results repeatable.

The two MPEG sources used are based on 24 minutes traces from the Bond movie "Goldfinger" and from an Asterix cartoon, which both have been made available by $O$. Rose at the Institute of Computer Science, University of Wuerzburg. The source for the Bond movie is implemented as a 65 state model and has a mean rate of $0.59 \mathrm{Mbps}$, while the Asterix cartoon has 52 states and a mean rate of $0.51 \mathrm{Mbps}$. Thereby we are able to multiplex both MPEG models in one STG without exceeding the upper limits for what the equipment can handle.

The on/off data source used in the experiments has a peak rate of $7.78 \mathrm{Mbps}$, a mean rate of $3.89 \mathrm{Mbps}$ and a mean on- and off duration of $50 \mathrm{msec}$. This source 
represents a high bandwidth source which is not so bursty. The duration in both onand off states is exponentially distributed and mutually independent (traditional 2state Markov source).

\subsection{Experimentally derived boundaries}

\subsubsection{Experimental configuration}

Figure 2 shows the experimental configuration involving a Cisco LS1010 ATM switch and a test instrument called ATM-100 which gives the possibility to generate and analyse quite general random traffic. The ATM-100 is equipped with two STGs (Helvic, 1993) which generate the artificial MPEG and the on/off data traffic. Due to hardware constraints in the traffic generators a pacing rate function has been used to limit the output port capacity to $37.44 \mathrm{Mbps}$, thereby reducing the number of sources required to adequately load the system. The traffic is multiplexed in an output port of the Cisco switch and the resulting traffic stream is analysed in the ATM-100, permitting cell loss and delay measurements.

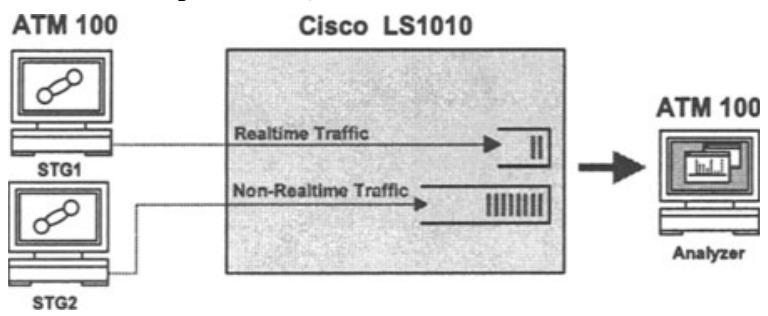

Figure 2: The experimental configuration

The buffer architecture of the Cisco switch implements delay priorities to protect the real-time ( $\mathrm{rt}$ ) traffic from influence of the non-real time (nrt) traffic. A short buffer has been used for the rt-traffic. For the nrt-traffic, which can tolerate longer delays, a longer buffer has been used. The service discipline is such that the rttraffic is served as long as there are cells in the short buffer. Only when the short buffer is empty, the nrt-traffic will be served.

\subsubsection{Measurement results}

In the first two sets of experiments a number of MPEG sources (Bond and Asterix) and on/off sources has been multiplexed in a large FIFO buffer of 1260 cells and a small one of 256 cells. In the third set of experiments the MPEG traffic has been multiplexed in a small high priority buffer ( 256 cells) while the on/off traffic is multiplexed in a larger low priority buffer (1260 cells). The number of sources has been varied to obtain a cell loss ratio (CLR) below, but as close to $10^{-4}$ as possible. By changing the traffic mix it has been possible to obtain the CAC admission boundary for each of the three cases. 
Figures 3 and 4 give the 2-dimensional CAC boundaries for Bond + on/off and Asterix + on/off. Experiments with a mix of the three types of sources have also been performed. From these measurements some points on the plane (the CAC boundary of MPEG-Bond + MPEG-Asterix + on/off) have been derived. Figure 5 gives the three-dimensional admission boundary for the non-priority case with a small buffer. The three-dimensional CAC boundary when delay priorities are used, can be found in Figure 6.

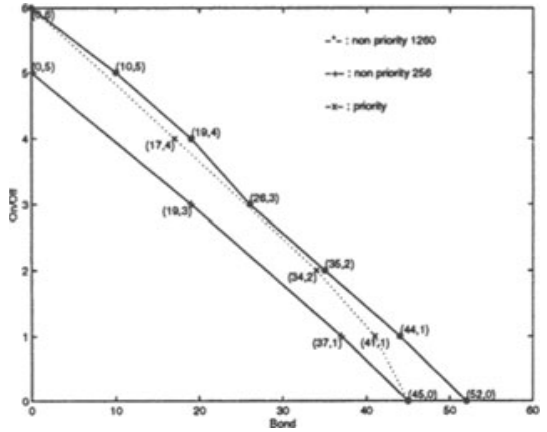

Figure 3: CAC boundaries for Bond + on/off.

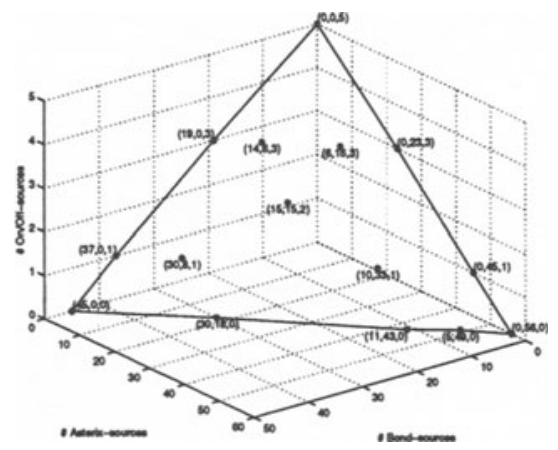

Figure 5: CAC boundary for the nonpriority case with buffer $=256$ cells.

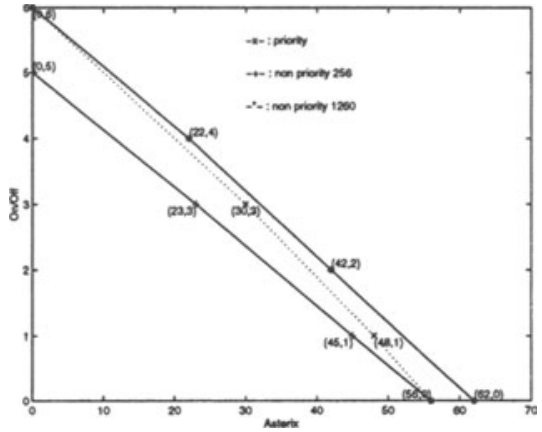

Figure 4: CAC boundaries for Asterix + on/off.

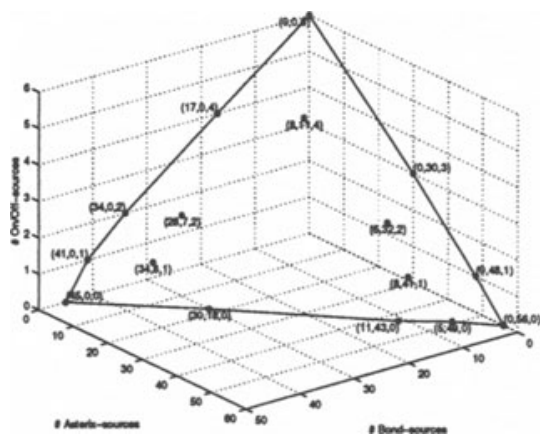

Figure 6: CAC boundary for the delay priority case.

In the non-priority case, the two dimensional boundaries are close to linear. The small deviations from linear are due to general uncertainties which exist in any measurement. This linearity supports the feasibility of the concept of effective bandwidth (Kelly, 1991). As expected, we can allow more MPEG sources in the delay priority case than when we use a small common buffer. Almost the same admission boundaries as in the non-priority case with large buffer come out, except that the number of MPEG sources that can be supported is less. The reason is that in the delay priority case the multiplexing of MPEG takes place in a buffer of size 256 while in the non-priority case the size of the buffer is almost 5 times larger and 
when no or only a small amount of on/off traffic is present this large pool can be used by the MPEG traffic. This of course at the expense of larger delay and larger variations in the delay.

\subsubsection{Simulation results}

Some aspects related with the STG have to be taken into account before comparing experimental and simulation results. The STGs have some hardware limitations which can make the implemented Markov models slightly different from the exact Markov models:

- only transition probability values in integer multiples of 1/256 are possible

- the peak rate must divide the link rate such that the interarrival time between cells in a given state is always the same integer number of slots.

The simulation tool used can provide any transition probability value as well as any peak bit rate over a period of observation. Depending on the number of sources generated, these slight differences may become important. They give rise to differences in the mean bit rate (in Mbps) for the MPEG sources (with a 95\% confidence level measured on 30 independent sources): Bond: 0.59221 (STG) vs. $0.59764 \pm 0.003$ (simulator) and Asterix: 0.51318 (STG) vs. $0.54943 \pm 0.002$ (simulator). Thus, the exact model for Asterix sources generates $0.03625 \mathrm{Mbps}$ more than the implemented model, i.e. for a certain experimental point the CLR obtained by simulation can be worse depending on the number of Asterix sources used, since in a congestion situation small increases in load can lead to big differences in CLR. Nevertheless, in those points where Bond sources have more influence, negligible differences between experimental and simulated CLR should be seen. For all cases presented in the Tables 1, 2 and 3 a $90 \%$ confidence interval was used.

Table 1: Non-priority case with 256 cells buffer size

\begin{tabular}{lllll}
\hline Bond & Asterix & On/off & Experimental CLR $\left[x 10^{-4}\right]$ & Simulated CLR $\left[x 10^{-4}\right]$ \\
\hline 14 & 6 & 3 & 0.5024 & $0.8295 \pm 0.0891$ \\
30 & 8 & 1 & 0.5637 & $0.8172 \pm 0.1503$ \\
45 & 0 & 0 & 0.5001 & $0.5747 \pm 1.1058$ \\
19 & 0 & 3 & 0.6423 & $0.7780 \pm 0.1035$ \\
0 & 23 & 3 & 0.3759 & $1.0975 \pm 0.1020$ \\
30 & 18 & 0 & 0.6477 & $0.7412 \pm 0.1608$ \\
\hline
\end{tabular}

Table 2: Non-priority case with 1260 cells buffer size

\begin{tabular}{lllll}
\hline Bond & Asterix & On/off & Experimental CLR $\left[x 10^{-4}\right]$ & Simulated CLR $\left[x 10^{-4}\right]$ \\
\hline 0 & 0 & 6 & 0.3798 & $0.3532 \pm 0.1416$ \\
10 & 0 & 5 & 0.9742 & $0.9032 \pm 0.2498$ \\
52 & 0 & 0 & 0.3090 & $0.8954 \pm 0.0913$ \\
0 & 22 & 4 & 0.6150 & $2.2484 \pm 0.3441$ \\
\hline
\end{tabular}


Table 3: Priority case $256 / 1260$ cells buffer size

\begin{tabular}{|c|c|c|c|c|c|c|}
\hline \multirow[b]{2}{*}{ Bond } & \multicolumn{2}{|c|}{ Sources } & \multicolumn{2}{|c|}{$\begin{array}{l}\text { Experimental CLR } \\
{\left[\begin{array}{ll}x & \left.10^{-4}\right]\end{array}\right.}\end{array}$} & \multicolumn{2}{|c|}{ Simulated CLR $\left[\begin{array}{ll}x & 10^{-4}\end{array}\right]$} \\
\hline & Asterix & On/off & $\begin{array}{l}\text { Bond- } \\
\text { Asterix }\end{array}$ & On/off & Bond-Asterix & On/off \\
\hline$\overline{0}$ & 30 & 3 & 0.0 & 0.6831 & 0.0 & $1.9 \pm 0.12$ \\
\hline 17 & 0 & 4 & 0.0 & 0.3230 & 0.0 & $0.72 \pm 0.19$ \\
\hline 8 & 11 & 4 & 0.0 & 0.8740 & 0.0 & $0.91 \pm 0.05$ \\
\hline 34 & 8 & 1 & 0.0134 & 0.8700 & $0.0225 \pm 0.0109$ & $1.11 \pm 1.01$ \\
\hline
\end{tabular}

\section{REAL MPEG TRACES AS INPUT TRAFFIC}

To evaluate the accuracy of the artificial MPEG sources used in the experiments, a multiplexing scenario has been simulated where traces of the real MPEG sources are mixed with on/off traffic. In order to obtain a sufficient number of MPEG sources a video sequence was chopped into a number of equally sized subsequences implying that it is possible to multiplex a high number of MPEG sources on the expense of a shorter simulation time. To avoid dependence between consecutive pieces of the chopped video traces there is a limit on the number of pieces a video sequence can be chopped into. We have considered one minute as a desirable minimum and 20 seconds as an absolute minimum. Due to the periodic nature of the MPEG coding, multiplexing experiments are highly dependent on how the actual phasing between the MPEG sources is. To obtain random phasing between the MPEG sources, a relatively large number of simulation runs needs to be performed.

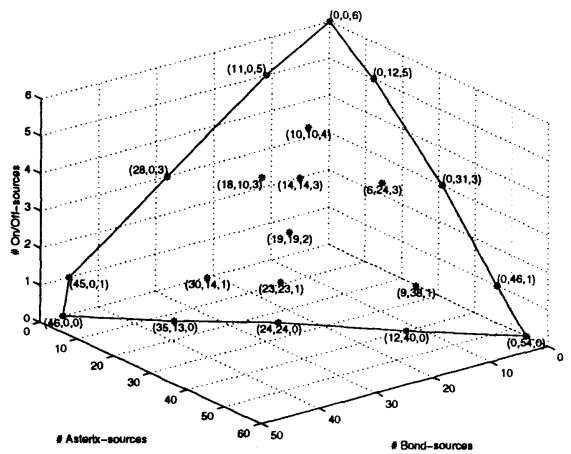

Figure 7: CAC boundary for the delay priority case

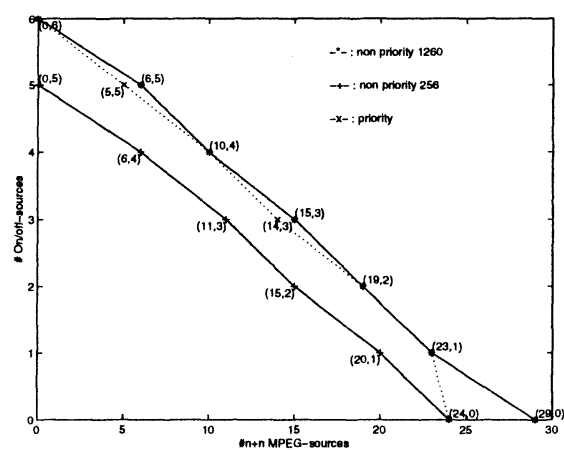

Figure 8: CAC boundaries for MPEG + on/off

Figure 7 shows the three dimensional admission boundary with a target CLR of $10^{-4}$ for the delay priority case. The results don't differ in a significant way from the experimental results which were based on the artificial MPEG sources. With a single exception, there is a good agreement between the CLR obtained in the trace 
driven simulations and the experimental results. Confidence intervals are quite big which is due to the tremendous influence the phasing of the individual MPEG sources has on the multiplexing buffer. Figure 8 shows a two dimensional admission boundary where we have constrained the number of Asterix and Bond sources to be equal. The boundaries have small discrepancies from linear which is due to the large confidence intervals in the simulations. However, the results do indicate that the admission set in the delay priority case , as a first approximation, can be determined as the intersection of the admission boundary in the non-priority case with large buffer and both types of traffic and the set determined by the high priority traffic multiplexed in the small buffer alone.

\section{ANALYTICALLY DERIVED BOUNDARIES}

A $10^{-4} \mathrm{CAC}$ boundary is derived analytically for the non-priority case with small buffer. By a straightforward discretisation of time, the Bond and Asterix STG sources are modelled as D-BMAPs (discrete-time batch Markovian arrival processes). To handle the multiplexing without a state space explosion, the circulant matching method of Hwang and Li (Hwang, 1995) is adapted for D-BMAPs by which an accurate approximation of the superposition process without too many states is made possible. The determination of cell loss is then reduced to the solution of a $\mathrm{D}-\mathrm{BMAP} / \mathrm{D} / 1 / \mathrm{K}+1$ queue.

\subsection{The discrete-time batch Markovian arrival process}

A discrete-time batch Markovian arrival process (D-BMAP) $(\mathbf{D})_{\mathrm{n} \geq 0}$ is defined as follows: the matrix $\mathbf{D}=\sum_{n=0}^{\infty} \mathbf{D}_{\mathrm{n}}$ is the transition matrix of a discrete-time finite Markov chain. Suppose that at time $k$ this chain is in some state $i$. At the next time instant $k+1$, there occurs a transition to another state $j$, with probability $(\mathbf{D})_{i, j}$ at which a batch arrival may or may not occur. The matrix $\mathbf{D}_{0}$ governs transitions that correspond to no arrivals while the matrices $\mathrm{D}_{n}, n \geq 1$, govern transitions that correspond to arrivals of batches of size $n$. For more details and properties, see (Blondia, 1993).

\subsection{A method to approximate the superposition of D-BMAPs}

Since the exact analytical description of the superposition of D-BMAPs, which is again a D-BMAP, has a state space size which is non tractable from a numerical point of view, it is necessary to replace the exact arrival process by another, but simpler process, which matches the exact one as close as possible for some important statistical functions. In (Hwang, 1995), it is proposed to replace the superposition of Markov modulated Poisson processes (MMPP) by a MMPP with a special structure, called circulant modulated Poisson process (CMPP). This process 
has a completely different Markovian structure as the superposition, but approximates two important statistical functions of the input rate process: the cumulative distribution representing the stationary statistics and the autocorrelation function in the time domain or equivalently the power spectral function in the frequency domain representing the second order statistics. Since our interest is in the superposition of D-BMAPs, the method is adapted for it and a circulant D-BMAP is used to replace the superposition. A circulant D-BMAP $\left(\mathbf{Q}_{k}\right)_{k \geq 0}$ of dimension $\mathrm{N}$ is defined by $\left(\mathbf{Q}_{\mathrm{k}}\right)_{i, j}=\mathrm{a}_{(\mathrm{j}-\mathrm{i}) \operatorname{modN}} \frac{\left(\gamma_{i}\right)^{k} e^{-\gamma_{i}}}{k !}$. The circulant D-BMAP is thus completely determined by $\mathbf{a}$, the first row of $\mathbf{Q}$ and a rate vector $\boldsymbol{\gamma}$.

The different steps in the procedure to obtain a circulant D-BMAP $(\mathbf{Q}, \gamma)$ as an approximation for the superposition of $M$ independent D-BMAPs $\left(\mathbf{D}_{k}^{(i)}\right)_{k \geq 0}$, and the extension of this method for periodic transition matrices are described in (Spaey, 1997). The steps are based on the analogous steps in the matching procedure described in (Hwang, 1995).

\subsection{Modelling of the MPEG and on/off sources as D-BMAPs}

The method developed by Helvik (Helvic, 1996) to generate the MPEG traffic source model used in the experiments gives us the following data as output:

- a periodic transition matrix which describes the transition probabilities between the different states of the model. The state sojourn time is $45 \mathrm{msec}$ for all states (a frame duration)

- a table with a load (expressed in bits per frame) for each state which is generated while being in that state

A D-BMAP $(\mathbf{D})_{\mathrm{n} \geq 0}$ can be gathered from this data by using the given transition matrix as the transition matrix D for the D-BMAP, and by transforming the loads in the load table into number of cells per frame. If this gives $\mathrm{x}$ cells per frame for state $i$, define then $\forall j:\left(\mathbf{D}_{\mathbf{x}}\right)_{i, j}:=(\mathbf{D})_{i, j},\left(\mathbf{D}_{\mathbf{y}}\right)_{i, j}:=0 \forall \mathrm{y} \neq \mathrm{x}$. Application of the method for the superposition of D-BMAPs on the Bond, Asterix or a combination of those sources, gives a new D-BMAP $(\mathbf{Q}, \gamma)$ which is an approximation for the superposition. The underlying time unit is still a frame length of $45 \mathrm{msec}$. Since this D-BMAP has to be used as input for a single server queue with a constant service time which equals the time needed to put one cell onto the outgoing link $(=1$ slot $),(\mathbf{Q}, \gamma)$ is transformed into a D-BMAP $(\hat{\mathbf{Q}}, \hat{\gamma})$ with one slot as the underlying time unit, supposing that the number of slots for being in a state is geometrically distributed with mean $x$, where $x$ is the number of slots in a frame duration.

The on/off sources used in the experiments have the following characteristics:

- in the on state, data is generated at $7.78 \mathrm{Mbps}$,

- the mean sojourntime in on and off state is $50 \mathrm{msec}$

A D-MAP for this kind of sources is generated by supposing again that the number of slots for being in a state is geometrically distributed, with mean $x$, where $x$ is 
now the number of slots in $50 \mathrm{msec}$ and by asuming that while the source is in the on state, the probability of generating a cell in a slot is $p$. To describe the superposition of $M$ independent on/off sources, the transition matrix $S$ of the number of sources which are in the on state is composed:

$(\mathbf{S})_{i, j}=\sum_{k=\max (0, i+j-M)}^{\min (i, j)}(1-\alpha)^{M-i-j+2 k} \alpha^{i+j-2 k}\left(\begin{array}{c}i \\ k\end{array}\right)\left(\begin{array}{c}M-i \\ j-k\end{array}\right)$ with $\alpha=\frac{1}{x}$

Since a source which is in the on state generates a cell in a slot with probability $p, k$ sources in the on state generate $t(t \in\{0, \ldots, k\})$ cells in a slot with probability $\left(\begin{array}{c}k \\ t\end{array}\right) p^{t}(1-p)^{k-t}$. From this, the matrices $\mathbf{S}_{0}, \ldots, \mathbf{S}_{\mathrm{M}}$ can be composed.

For the superposition of a number of MPEG sources based on the Helvik model and some on/off sources, the new D-BMAP $\left(\mathbf{T}_{n}\right)_{n \geq 0}$ is composed as the superposition of $\left(\hat{\mathbf{Q}}_{n}\right)_{n \geq 0}$ and $\left(\mathbf{S}_{n}\right)_{n \in\{0, \ldots, M)}$.

\subsection{The queueing model and numerical results}

The multiplexer whose input consists of the superposition of Bond, and/or Asterix and/or on/off sources is modelled as a discrete-time D-BMAP/D/1/K+1 queue. The input D-BMAPs are obtained by the approximation method, $K+1$ is the systemlength and the servicetime equals one timeslot. In (Blondia,1992), the $\mathrm{D}-\mathrm{BMAP} / \mathrm{D} / 1 / \mathrm{K}+1$ queue was solved analytically and these results are applied to obtain the CLR.

All the results are obtained for a queue length of 100 cells and an outgoing link of 37.44 Mbps. This implies that one time slot equals $11.325 \mu \mathrm{sec}$. By transforming bits to number of cells, it is assumed that a cell of 53 bytes can contain 48 bytes of data. Figure 9 shows the analytical derived $10^{-4} \mathrm{CAC}$ boundary for the non-priority case. As in the experiments, for a mix of two types of sources, the admission boundaries are close to linear. If the obtained points are compared with experimental points (see Table 4), which is not possible for all points since only a few experiments are performed with a buffer size of 100 cells, it is seen that all the analytical results are more conservative than the experimental results.

Table 4: Comparison between analytically and experimentally obtained results

\begin{tabular}{llllll} 
Experimental points & $(44,0)$ & $(30,17)$ & $(20,29)$ & $(10,42)$ & $(0,55)$ \\
Analytical points & $(43,0)$ & $(30,14)$ & $(20,26)$ & $(10,38)$ & $(0,51)$ \\
\hline
\end{tabular}

Although the analytical obtained results are compared here with experimental CAC results, the matching method is basically a method used for avoiding the state space explosion when multiplexing D-BMAPs. Since the CAC experiments are based on multiplexing, these experiments were used to test the behaviour of the analytical approximation method for multiplexing D-BMAPs, with the cell loss ratio as used measure of performance. 


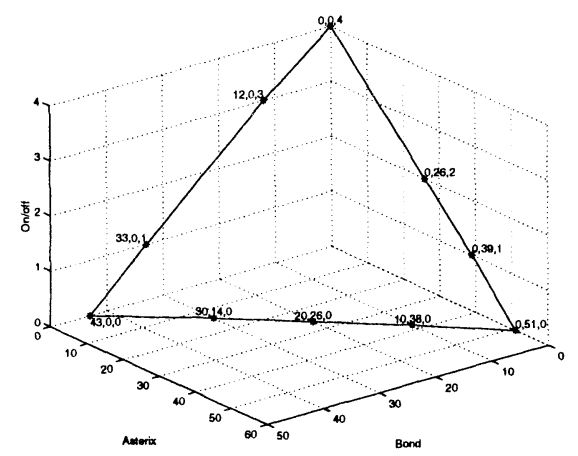

Figure 9: Analytically derived CAC boundary for a non-priority buffer of 100 cells

\section{CONCLUSIONS}

Extensive multiplexing experiments using model-based traffic generators have been carried out at the EXPERT ATM testbed in Basel to obtain CAC boundaries for traffic scenarios involving MPEG video and data traffic. Both FIFO as well as strict delay priority scheduling schemes have been studied, and the experimental results have been compared with simulation results and results based on an analytical method.

The flexibility of software simulations has been exploited to investigate any possible measurement error introduced due to differences between the derived MPEG models and MPEG models as implemented in the traffic generators. The simulation results support the validity of the traffic source implementation.

Trace driven simulations have been performed to compare the multiplexing behaviour of the MPEG sources with the video traces on which the artificial MPEG sources are based. Although this procedure is associated with some difficulties due to the dependency on phasing between individual traces, some evidence is given to the validity of the chosen MPEG modelling approach.

Furthermore, the non-priority case with a small buffer has been analysed using analytically derived boundaries based on D-BMAP models of the traffic sources. The matching method based on circulants has been applied to approximate the superposition of D-BMAP models by another D-BMAP model with much smaller state space thus making a queueing analysis feasible.

By combining results from hardware experiments, software simulations and analytical methods we have demonstrated a coherent approach for investigating multiplexed video and data traffic. Application of these methods can be used for developing efficient network resource management schemes.

Acknowledgements: The achievements being made within the ACTS project EXPERT are only possible with the conscientious co-operation of all partners and the support of the Commission of the European Union. The contribution of all these players is therefore hereby gratefully acknowledged. We also acknowledge O. Rose 
of the University of Wuerzburg for providing us the video traces, as well as $\mathrm{H}$. Christiansen of the Technical University of Denmark for his trace driven program with which the results of section 3 have been derived.

\section{REFERENCES}

Beran, J., Sherman, R., Taqqua, M. and Willinger, W. (1995) Variable bit rate video traffic and long range dependence, IEEE Trans. on Comm., 43, 1566-79.

Blondia, C. and Casals, O. (1992) Statistical multiplexing of VBR sources: A matrix analytical approach, Performance Evaluation, 16, 5-20.

Blondia, C. (1993) A discrete-time batch Markovian arrival process as B-ISDN traffic model, Belgian Journal of Operations Research, Statistics and Computer Science, 32, 3-23.

Draft International Standard ISO/IEC DIS 13818-2 (1993a) Generic coding of moving pictures and associated audio Part 2 Video.

Helvic, B., Melteig, O. and Morland, L. (1993) The synthesized traffic generator: objectives, design and capabilities, Proceedings of the IBCN\&S.

Helvik, B. (1996) MPEG source type models for the STG (Synthesized Traffic Generator), SINTEF report STF40 A96016.

Hwang, C.L. and Li, S.Q. (1995) On the convergence of traffic measurement and queueing analysis: A Statistical MAtch Queueing (SMAQ) tool, Proc. of IEEE Infocom 95, 602-12.

International Standard ISO/IEC DIS 13818-2 (1993b) Coding of moving pictures and associated audio for digital storage media up to $1.5 \mathrm{Mbit} / \mathrm{s}$ Part 2 Video.

Kelly, F.P. (1991) Effective bandwidth at multi-class queues, Queueing Systems, 9, 5-16.

Le Gall, D. (1991) MPEG: A video compression scheme standard for multimedia applications, Comm. ACM, 34(4), 46-58.

Leland, W.E., Taqqu, M.S., Willinger, W. and Wilson, D.V. (1994) On the selfsimilar nature of Ethernet traffic, IEEE/ACM Trans. on Networking, 2, 1-15.

Maglaris, B., Anastassiou D., Sen, P., Karlsson, G. and Robbins, J.D. (1988) Performance models of statistical multiplexing in packet video multiplexers. IEEE Trans. on Comm, 36, 834-44.

Ramamurthy, G. and Sengupta, B. (1992) Modelling and analysis of a variable bit rate video multiplexer, IEEE Infocom 92.

Reininger, D., Melamed, B. and Raychaudhuri, D. (1994) Variable bit rate MPEG video: characteristics, modelling and multiplexing. ITC-14, 295-306.

Roberts, J., Mocci, U. and Virtamo, J. (ed.) (1996) Broadband network traffic, Final Report of Action Cost 242, Springer Verlag.

Rose, O. (1997) Discrete time analysis of finite buffer with VBR MPEG video traffic input, ITC-15, 413-22.

Spaey, K. and Blondia, C. (1997) Circulant matching method for multiplexing ATM traffic applied to video sources, submitted for publication. 